\title{
Implicit Finite Difference Method for Fractional Wave Equation with Dirichlet and Fractional Boundary Conditions
}

\section{Shaomei Fang ${ }^{1,2, a}$, Wenjie Huang ${ }^{1, b}$, Changping Xie ${ }^{1, c,{ }^{*}}$, Yingshu Zhang ${ }^{1, d}$, Jinyan $\mathrm{Li}^{1, \mathrm{e}}$ and Zhenfu Cai ${ }^{1, \mathrm{f}}$}

${ }^{1}$ College of Mathematics and Informatics, South China Agricultural University, Guangzhou 510642 , People's Republic of China

${ }^{2}$ School of Mathematics and statistics, Yangtze Normal University, Chongqing 408100, People's Republic of China

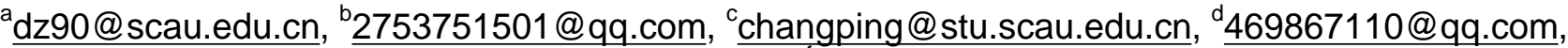
'duduwoxing@126.com, 229430694@qq.com

${ }^{*}$ Corresponding author

Keywords: Fractional wave equation; Riemann-Liouville fractional derivative; Fractional boundary conditions; Finite difference method; Consistency and stability

Abstract. In this paper, the implicit finite difference method is developed for the fractional wave equation with Dirichlet and fractional boundary conditions. The consistency and stability of the method are strictly proved by the Gerschgorin theorem and mathematical induction. Numerical examples show the accuracy and efficiency of the scheme and coincide with the theoretical analysis.

\section{Introduction}

Fractional differential equations are generalizations of integer-order differential equations, which have been used in many models, such as fractional diffusion equations describing anomalous diffusion [1,2] and continuous-time finance problems [3], fractional Fokker-Planck equations describing Lévy stable processes [4,5] and porous media [6], and fractional wave equations describing chiral media [7-9], waveguides [10], reflection and scattering problems [11]. As most of fractional differential equations do not have explicit analytical solutions, research on numerical methods of fractional differential equations become critical.

In this paper, we consider the fractional wave equation with the the left-sided fractional spatial derivative as follows

$$
\frac{\partial^{2} u(x, t)}{\partial t^{2}}=c(x, t) \frac{\partial^{\alpha} u(x, t)}{\partial_{+} x^{\alpha}}+d(x, t), 0<x<L, 0<t<T,
$$

subject to the boundary and initial conditions

$$
\begin{aligned}
& u(0, t)=0, \gamma u(L, t)+\left(c(x, t) \frac{\partial^{\alpha-1} u(x, t)}{\partial_{+} x^{\alpha-1}}\right)_{x=L}=v(t), 0<t<T, \\
& u(x, 0)=p^{0}(x),\left.\frac{\partial u(x, t)}{\partial t}\right|_{t=0}=p^{1}(x), \quad 0<x<L,
\end{aligned}
$$

where the parameter $\alpha$ describes the fractional order of spatial derivatives with $1<\alpha<2$. Coefficient function $c(x, t)$ is positive, $d(x, t)$ refers to a source term, $\gamma=0$ corresponds to a fractional Neumann boundary condition, and $\gamma>0$ corresponds to a fractional Robin boundary condition. 
The space fractional derivatives are defined as the Riemann-Liouville fractional derivative [12-14]

$$
\frac{\partial^{\beta} u(x)}{\partial_{+} x^{\beta}}=\frac{1}{\Gamma(n-\beta)} \frac{d^{n}}{d x^{n}} \int_{0}^{x} \frac{u(s)}{(x-s)^{\beta+1-n}} d s,
$$

where $n$ is an integer, and $n-1<\beta \leq n$. When $\beta$ is an integer, (4) gives the standard integer derivative.

Recently, Jia and Wang [15] developed a fast finite difference method for space-fractional diffusion equations with fractional derivative boundary conditions in one space dimension. Guo et al. [16] processed an implicit finite difference method for a one-dimensional fractional percolation equation with the Dirichlet and fractional boundary conditions. To our knowledge, implict finite difference method for fractional wave equation with the Dirichlet and fractional boundary conditions is still limited. This motivate us to examine a numerical approach for it.

The rest of the paper is organized as follows. In Section 2, we construct an implicit finite difference scheme and study its consistency. The stabilty of the scheme is proved in Section 3. In Section 4, we carry out numerical examples to check the accuracy and efficiency of the proposed scheme. Finally, we draw our conclusions in Section 5.

\section{Implicit finite difference method and its consistency}

In this section, we construct an implicit finite difference scheme and study the consistency for (1-3).

For the numerical approximation scheme, Let $t_{m}=m \Delta t(m=0,1,2, \mathrm{~L}, M), x_{i}=i h(i=0,1,2, \mathrm{~L}, N)$

to be the temporal partition and the spatial partition, respectively, where $M$ and $N$ are positive integers. Then time grid size is $\Delta t=T / M$ and space grid size is $h=L / N$. Denote the exact and numerical solution at the mesh point $\left(x_{i}, t_{m}\right)$ by $U_{i}^{m}$ and $u_{i}^{m}$, respectively. Define

$$
c_{i}^{m}=c\left(x_{i}, t_{m}\right), d_{i}^{m}=d\left(x_{i}, t_{m}\right), v^{m}=v\left(t_{m}\right), p_{i}^{0}=p^{0}\left(x_{i}\right), p_{i}^{1}=p^{1}\left(x_{i}\right),
$$

and initial and the Dirichlet boundary conditions

$$
u_{i}^{0}=p_{i}^{0}, u_{0}^{m}=0 .
$$

According to [17], the left-sided fractional spatial derivative can be approximated by the shifted left Grünwald-Letnikov derivative

$$
\left.\frac{\partial^{\alpha} u(x, t)}{\partial_{+} x^{\alpha}}\right|_{\left(x_{i}, t_{m+1}\right)}=\frac{1}{h^{\alpha}} \sum_{j=0}^{i} g_{j}^{(\alpha)} U_{i-j+1}^{m+1}+O(h)
$$

where $1 \leq i \leq N-1,0 \leq m \leq M-1$, and $g_{j}^{(\alpha)}=(-1)^{j}\left(\begin{array}{c}\alpha \\ j\end{array}\right)$ are the fractional binomial coefficients.

To approximate (1) we use the backward Euler difference scheme for the second order time derivative

$$
\left.\frac{\partial^{2} u(x, t)}{\partial t^{2}}\right|_{\left(x_{i}, t_{m+1}\right)}=\frac{U_{i}^{m+1}-2 U_{i}^{m}+U_{i}^{m-1}}{(\Delta t)^{2}}+O(\Delta t)
$$

where $1 \leq i \leq N-1,1 \leq m \leq M-1$.

And the left-sided fractional spatial derivative of the order $\alpha-1$ in (2) can be discretized by the standard left Grünwald-Letnikov fractional derivative [17] as 


$$
\left.\frac{\partial^{\alpha-1} u(x, t)}{\partial_{+} x^{\alpha-1}}\right|_{\left(L, t_{m+1}\right)}=\frac{1}{h^{\alpha-1}} \sum_{j=0}^{N-1} g_{j}^{(\alpha-1)} U_{N-j}^{m+1}+O(h),
$$

where $0 \leq m \leq M-1$.

The first order time derivative in (3) can be approximated by the forward Euler difference scheme

$$
\left.\frac{\partial u(x, t)}{\partial t}\right|_{\left(x_{i}, 0\right)}=\frac{U_{i}^{1}-U_{i}^{0}}{\Delta t}+O(\Delta t),
$$

where $1 \leq i \leq N-1$.

Therefore, an implicit finite difference scheme can be expressed as follows:

For $m=0$,

$$
\begin{aligned}
& p_{i}^{1}=\frac{u_{i}^{1}-u_{i}^{0}}{\Delta t}, 1 \leq i \leq N-1, \\
& u_{0}^{1}=0, \gamma u_{N}^{1}+\frac{c_{N}^{1}}{h^{\alpha-1}} \sum_{j=0}^{N-1} g_{j}^{(\alpha-1)} u_{N-j}^{1}=v^{1},
\end{aligned}
$$

For $1 \leq m \leq M-1$,

$$
\begin{aligned}
& \frac{u_{i}^{m+1}-2 u_{i}^{m}+u_{i}^{m-1}}{(\Delta t)^{2}}=\frac{c_{i}^{m+1}}{h^{\alpha}} \sum_{j=0}^{i} g_{j}^{(\alpha)} u_{i-j+1}^{m+1}+d_{i}^{m+1}, 1 \leq i \leq N-1, \\
& u_{0}^{m+1}=0, \gamma u_{N}^{m+1}+\frac{c_{N}^{m+1}}{h^{\alpha-1}} \sum_{j=0}^{N-1} g_{j}^{(\alpha-1)} u_{N-j}^{m+1}=v^{m+1},
\end{aligned}
$$

Denote the local truncation error by $R_{i}^{m}$ for $1 \leq i \leq N$. It follows from (9)-(12) that

$$
\begin{aligned}
& R_{i}^{1}=p_{i}^{1}-\frac{U_{i}^{1}-U_{i}^{0}}{\Delta t}=O(\Delta t), 1 \leq i \leq N-1, \\
& R_{i}^{m+1}=\frac{U_{i}^{m+1}-2 U_{i}^{m}+U_{i}^{m-1}}{(\Delta t)^{2}}-\frac{c_{i}^{m+1}}{h^{\alpha}} \sum_{j=0}^{i} g_{j}^{(\alpha)} U_{i-j+1}^{m+1}-d_{i}^{m+1}=O(\Delta t)+O(h), 1 \leq i \leq N-1,1 \leq m \leq M-1, \\
& R_{N}^{m+1}=\gamma U_{N}^{m+1}+\frac{c_{N}^{m+1}}{h^{\alpha-1}} \sum_{j=0}^{N-1} g_{j}^{(\alpha-1)} U_{N-j}^{m+1}-v^{m+1}-O(h), 0 \leq m \leq M-1 .
\end{aligned}
$$

This implies the consistency of the implicit finite difference scheme difined by (9)-(12).

\section{Stability analysis}

In this section, we discuss the stability and convergence of the numerical method (9)-(12).

Let $r=\frac{(\Delta t)^{2}}{h^{\alpha}}$. One can rewrite (11) in the form:

$$
u_{i}^{m+1}-r c_{i}^{m+1} \sum_{j=0}^{i} g_{i-j+1}^{(\alpha)} u_{j}^{m+1}=2 u_{i}^{m}-u_{i}^{m-1}+(\Delta t)^{2} d_{i}^{m+1}, 1 \leq i \leq N-1,
$$

where $1 \leq m \leq M-1$. Then the implicit finite difference schemes (9)-(12) can be presented as the matrix form as follows:

$$
A^{(1)} U^{1}=P^{1}+P^{0},
$$




$$
A^{(2)} U^{m+1}=2 Q^{m}-Q^{m-1}+D^{m+1}, 1 \leq m \leq M-1,
$$

where

$$
\begin{aligned}
& U^{m}=\left(u_{1}^{m}, u_{1}^{m}, \mathrm{~L}, u_{N}^{m}\right)^{T}, \\
& P^{1}=\left(\Delta t p_{1}^{1}, \Delta t p_{1}^{1}, \mathrm{~L}, \Delta t p_{N-1}^{1}, 0\right)^{T}, \\
& P^{0}=\left(p_{1}^{0}, p_{1}^{0}, \mathrm{~L}, p_{N-1}^{0}, h^{\alpha-1} v^{1}\right)^{T}, \\
& Q^{m}=\left(u_{1}^{m}, u_{1}^{m}, \mathrm{~L}, u_{N-1,0}^{m}\right)^{T}, \\
& D^{m}=\left((\Delta t)^{2} d_{1}^{m},(\Delta t)^{2} d_{1}^{m}, \mathrm{~L},(\Delta t)^{2} d_{N-1}^{m}, h^{\alpha-1} v^{m}\right)^{T},
\end{aligned}
$$

and the coefficient matrix $A^{(1)}, A^{(2)}$ and their entries are

$$
\begin{aligned}
& A^{(1)}=\left(A_{i, j}^{(1)}\right)_{i, j=1}^{N}=\left\{\begin{array}{l}
1, \quad 1 \leq i=j \leq N-1, \\
0, \quad i \neq j, 1 \leq j \leq N, 1 \leq i \leq N-1, \\
c_{N}^{1} g_{N-j}^{(\alpha-1)}, 1 \leq j \leq N-1, i=N, \\
h^{\alpha-1} \gamma+c_{N}^{1} g_{0}^{(\alpha-1)}, i=j=N .
\end{array}\right. \\
& A^{(2)}=\left(A_{i, j}^{(2)}\right)_{i, j=1}^{N}=\left\{\begin{array}{l}
-r c_{i}^{m+1} g_{i-j+1}^{(\alpha)}, \quad 1 \leq j<i, 1 \leq i \leq N-1, \\
1-r c_{i}^{m+1} g_{1}^{(\alpha)}, \quad 1 \leq j=i \leq N-1, \\
-r c_{i}^{m+1} g_{0}^{(\alpha)}, j=i+1,1 \leq i \leq N-1, \\
0, i+2 \leq j \leq N, 1 \leq i \leq N-2, \\
c_{N}^{m+1} g_{N-j}^{(\alpha-1)}, 1 \leq j \leq N-1, i=N, \\
h^{\alpha-1} \gamma+c_{N}^{m+1} g_{0}^{(\alpha-1)}, i=i=N .
\end{array}\right.
\end{aligned}
$$

In the following, we present some lemmas which are used later in this paper. First, we list some properties of the alternating fractional binomial coefficients [14].

Lemma 1. Let $\beta, \beta_{1}$, and $\beta_{2}$ be positive real numbers, and the integer $n \geq 1$. We have

(i) $g_{0}^{(\beta)}=1, g_{j}^{(\beta)}=\left(1-\frac{\beta+1}{j}\right) g_{j-1}^{(\beta)}$ for $j \geq 1$;

(ii) $g_{1}^{(\beta)}<g_{2}^{(\beta)}<\mathrm{L}<0, \sum_{j=0}^{n} g_{j}^{(\beta)}>0$ for $0<\beta<1$;

(iii) $g_{2}^{(\beta)}>g_{3}^{(\beta)}>\mathrm{L}>0, \sum_{j=0}^{n} g_{j}^{(\beta)}<0$ for $1<\beta<2$;

(iv) $\sum_{j=0}^{n} g_{j}^{(\beta)}=(-1)\left(\begin{array}{c}\beta-1 \\ n\end{array}\right)^{n}$;

(v) $\sum_{j=0}^{n} g_{j}^{\left(\beta_{1}\right)} g_{n-j}^{\left(\beta_{2}\right)}=g_{n}^{\left(\beta_{1}+\beta_{2}\right)}$.

Before giving the Lemma 2, we consider a equivalent form of the the finite difference scheme (18). Let us express the coefficient matrix $A^{(2)}$ defined by (20) in a block form as follows

$$
A^{(2)}=\left(\begin{array}{cc}
A_{N-1, N-1} & A_{N-1, N} \\
A_{N, N-1}^{T} & A_{N, N}^{(2)}
\end{array}\right),
$$


where

$$
A_{N-1, N-1}=\left(A^{(2)}\right)_{i, j=1}^{N-1}, A_{N-1, N}=\left(A_{i, N}^{(2)}\right)_{i=1}^{N-1}, A_{N, N-1}^{T}=\left(A_{N, j}^{(2)}\right)_{j=1}^{N-1} .
$$

Denote $\omega_{N}=\frac{r c_{N-1}^{m+1}}{c_{N}^{m+1}+h^{\alpha-1} \gamma}$. Multiply the $N$ th equation by $\omega_{N}$ and add to the $N-1$ th equation of the linear system (18). Then we get the following equivalent form of (18)

$$
A^{(3)} U^{m+1}=2 Q^{m}-Q^{m-1}+\bar{D}^{m+1}, 1 \leq m \leq M-1,
$$

where

$$
\begin{aligned}
& \bar{D}^{m}=\left((\Delta t)^{2} d_{1}^{m},(\Delta t)^{2} d_{1}^{m}, \mathrm{~L},(\Delta t)^{2} d_{N-1}^{m}+h^{\alpha-1} v^{m}, h^{\alpha-1} v^{m}\right)^{T}, \\
& A^{(3)}=\left(\begin{array}{cc}
\bar{A}_{N-1, N-1} & 0 \\
A_{N, N-1}^{T} & A_{N, N}^{(2)}
\end{array}\right), \\
& \bar{A}_{N-1, N-1}-I-r B, \\
& B=\left(B_{i, j}\right)_{i, j=1}^{N-1}=\left\{\begin{array}{l}
c_{i}^{m+1} g_{i-j+1}^{(\alpha)}, \quad 1 \leq j<i, 1 \leq i \leq N-2, \\
c_{i}^{m+1} g_{1}^{(\alpha)}, \quad 1 \leq j=i \leq N-2, \\
c_{i}^{m+1} g_{0}^{(\alpha)}, j=i+1,1 \leq i \leq N-2, \\
0, i+2 \leq j \leq N, 1 \leq i \leq N-3, \\
c_{N-1}^{m+1} c_{N}^{m+1}\left(g_{N-j}^{(\alpha-1)}-g_{N-j}^{(\alpha)}\right)+h^{\alpha-1} \gamma g_{N-j}^{(\alpha)}, \quad 1 \leq j \leq N-1, i=N-1,
\end{array}\right.
\end{aligned}
$$

Lemma 2. The spectral radius of the matrix $\left(\bar{A}_{N-1, N-1}\right)^{-1}$ is less than 1 .

Proof Our first goal is to show that the eigenvalues of the matrix $B$ have negative real parts. As $c_{i}^{m+1}>0$, and then by Lemma 1 , we have

$$
\begin{aligned}
& B_{i, j}>0, j \neq i, \\
& B_{i, j}<0, j=i,
\end{aligned}
$$

where $1 \leq i \leq N-1$.

When $1 \leq i \leq N-2$, we have

$$
B_{i, i}+\sum_{j=1, j \neq i}^{N-1}\left|B_{i, j}\right|=c_{i}^{m+1}\left(g_{1}^{(\alpha)}+\sum_{j=1}^{i-1} g_{i-j+1}^{(\alpha)}+g_{0}^{(\alpha)}\right)=c_{i}^{m+1} \sum_{j=0}^{i} g_{j}^{(\alpha)}<0,
$$

Similarly, when $i=N-1$, we obtain

$$
B_{N-1, N-1}+\sum_{j=1}^{N-2}\left|B_{N-1, j}\right|<0
$$

Then by Gerschgorin theorem [18], we have that all of the Greschgorin disks of the matrix $B$ are within the left half of the complex plane.

Next, from (27), we can clear know that the eigenvalues of the matrix $\bar{A}_{N-1, N-1}$ has a magnitude larger than 1 . Therefore, the spectral radius of the matrix $\left(\bar{A}_{N-1, N-1}\right)^{-1}$ is less than 1 . This completes the proof.

To discuss the stability of the numerical method, we denote 


$$
\varepsilon_{i}^{m}=u_{i}^{m}-\mathfrak{W}_{i}^{2}, 1 \leq i \leq N, 1 \leq m \leq M,
$$

and

$$
\varepsilon^{m}=\left(\varepsilon_{1}^{m}, \varepsilon_{2}^{m}, \mathrm{~L}, \varepsilon_{N}^{m}\right)^{T},\left\|\varepsilon^{m}\right\|_{\infty}=\max _{1 \leq i \leq N}\left|\varepsilon_{i}^{m}\right|,
$$

where $\mathscr{W}_{i}^{\prime}$ is the approximate solution of the difference scheme with the initial condition $\mathscr{O}_{i}$.

From the definition of the finite difference scheme, we have:

For $1 \leq i \leq N-1$,

$$
\begin{aligned}
& \varepsilon_{i}^{1}=\varepsilon_{i}^{0} \\
& \varepsilon_{i}^{m+1}-r c_{i}^{m+1} \sum_{j=0}^{i} g_{i-j+1}^{(\alpha)} \varepsilon_{j}^{m+1}=2 \varepsilon_{i}^{m}-\varepsilon_{i}^{m-1}, 1 \leq m \leq M-1 .
\end{aligned}
$$

For $i=N$,

$$
\left(h^{\alpha-1} \gamma+c_{N}^{m+1} g_{0}^{(\alpha-1)}\right) \varepsilon_{N}^{m+1}+c_{N}^{m+1} \sum_{j=1}^{N-1} g_{j}^{(\alpha-1)} \varepsilon_{N-j}^{m+1}=0,0 \leq m \leq M-1
$$

where $\varepsilon_{i}^{0}=0(m=0,1, \mathrm{~L} M)$.

For the stability of the numerical method, we have the following theorem.

Proposition 1. There exists a positive constant independent of $\Delta t$ and $h$ such that

$$
\left\|\varepsilon^{m}\right\|_{\infty} \leq C\left\|\varepsilon^{0}\right\|_{\infty}, 1 \leq m \leq M \text {. }
$$

Proof Let

$$
\zeta^{m}=\left(\varepsilon_{1}^{m}, \varepsilon_{2}^{m}, \mathrm{~L}, \varepsilon_{N-1}^{m}\right)^{T}, 0 \leq m \leq M .
$$

Then (33)-(35) can be rewritten as

$$
\begin{aligned}
& \zeta_{i}^{1}=\zeta_{i}^{0} \\
& \bar{A}_{N-1, N-1} \zeta^{m+1}=2 \zeta^{m}-\zeta^{m-1}, 1 \leq m \leq M-1 .
\end{aligned}
$$

For $i=N$,

$$
A_{N, N-1}^{T} \zeta^{m+1}+A_{N, N}^{(2)} \varepsilon_{N}^{m+1}=0,0 \leq m \leq M-1 .
$$

It follows from (39) that

$$
\left|\varepsilon_{N}^{m+1}\right|=\frac{\left|c_{N}^{m+1} \sum_{j=1}^{N-1} g_{j}^{(\alpha-1)} \varepsilon_{N-j}^{m+1}\right|}{\left|h^{\alpha-1} \gamma+c_{N}^{m+1} g_{0}^{(\alpha-1)}\right|} \leq \frac{c_{N}^{m+1} \sum_{j=1}^{N-1}\left|g_{j}^{(\alpha-1)}\right|\left|\varepsilon_{N-j}^{m+1}\right|}{\left|h^{\alpha-1} \gamma+c_{N}^{m+1} g_{0}^{(\alpha-1)}\right|}<\max _{1 \leq i \leq N-1}\left|\varepsilon_{i}^{m+1}\right| .
$$

This means that

$$
\left\|\varepsilon^{m+1}\right\|_{\infty}=\max _{1 \leq i \leq N}\left|\varepsilon_{i}^{m+1}\right|=\left\|\zeta^{m+1}\right\|_{\infty} .
$$

Then (37) implies that

$$
\left\|\varepsilon^{1}\right\|_{\infty}=\left\|\zeta^{1}\right\|_{\infty}=\left\|\zeta^{0}\right\|_{\infty}=\left\|\varepsilon^{0}\right\|_{\infty} .
$$


Suppose that $\left\|\varepsilon^{k}\right\|_{\infty} \leq C\left\|\varepsilon^{0}\right\|_{\infty}, k=2,3, \mathrm{~L}, m$, let $\left|\varepsilon_{l}^{m+1}\right|=\max _{1 \leq i \leq N}\left|\varepsilon_{i}^{m+1}\right|$.

Then by lemma 2, we have

$$
\left\|\varepsilon^{m+1}\right\|_{\infty}=\left|\varepsilon_{l}^{m+1}\right| \leq 2\left|\zeta^{m}\right|+\left|\zeta^{m-1}\right| \leq 2\left\|\zeta^{m}\right\|_{\infty}+\left\|\zeta^{m-1}\right\|_{\infty} \leq 3 C\left\|\zeta^{0}\right\|_{\infty}=3 C\left\|\varepsilon^{0}\right\|_{\infty}=C^{\prime}\left\|\varepsilon^{0}\right\|_{\infty} .
$$

Using mathematical induction, we complete the proof.

The following theorem can be obtained by above proposition [19-20].

Theorem 1. The implicit finite difference (17)-(18) is unconditional stable.

\section{Numerical example}

In this section, we consider the numerical solution of the fractional wave equation using the proposed scheme (9)-(12).

Example. We consider the following fractional wave equation

$$
\frac{\partial^{2} u(x, t)}{\partial t^{2}}=c(x, t) \frac{\partial^{\alpha} u(x, t)}{\partial_{+} x^{\alpha}}+d(x, t), \quad 0<x<1,0<t<1,
$$

with the fractional boundary conditions

$$
u(0, t)=0, \gamma u(1, t)+\left(c(x, t) \frac{\partial^{\alpha-1} u(x, t)}{\partial_{+} x^{\alpha-1}}\right)_{x=1}=v(t)=\exp (-t)\left(3 \gamma+\frac{6}{3-\alpha}\right),
$$

and initial conditions

$$
u(x, 0)=p^{0}(x)=3 x^{2},\left.\frac{\partial u(x, t)}{\partial t}\right|_{t=0}=p^{1}(x)=-3 x^{2}
$$

where $c(x, t)=\mathrm{T}(3-\alpha) x^{\alpha}, d(x, t)=-3 x^{2} \exp (-t)$.

The exact solution of this problem is given by

$$
u(x, t)=3 x^{2} \exp (-t) \text {. }
$$

A comparison between the exact and the numerical solutions using the proposed method with $\Delta t=h=1 / 40$ at $T=1$ is presented in Fig. 1. It can be seen that our numerical results are in excellent agreement with the exact solution both for the 145 fractional Neumann boundary condition $(\gamma=0)$ and Robin boundary condition $(\gamma=1)$.

Table 1 shows the maximum errors at the time $T=1$, where $h=10^{-4}$, a value small enough such that the space discretization errors are negligible while comparing with the time errors. It illustrates that the numerical method is temporally first-order accurate both for the fractional Neumann and Robin boundary condition. In Tables 2, with sufficiently small time step size $\Delta t=10^{-4}$, the convergence order in space of our schemes is also checked as first-order accurate. This implys the stability of the implicit finite difference scheme (9)-(12) proved in Theorem 1 and shows that the scheme are temporally and spatially first-order accurate. 


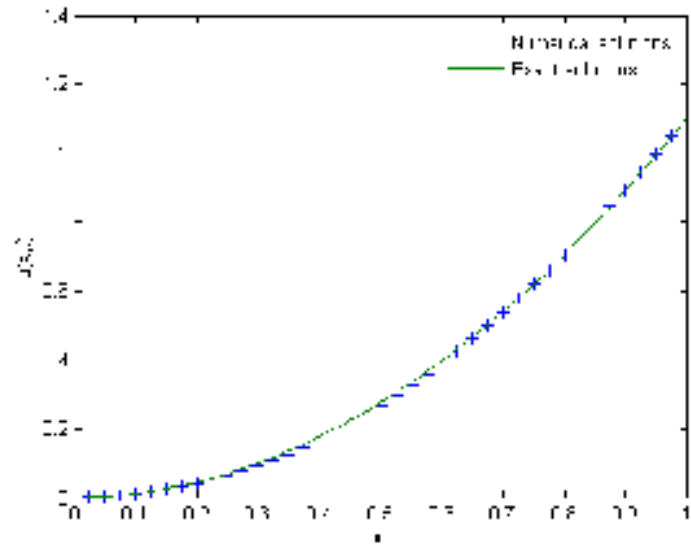

(a)

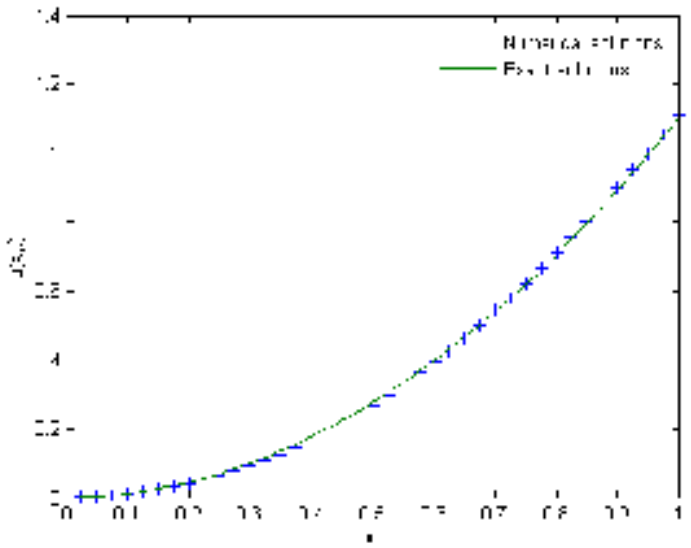

(b)

Fig.1 The comparisons of numerical solution and exact solution for $\alpha=1.9, \Delta t=h=1 / 40$ at the time $T=1$ (a) $\gamma=0$ (b) $\gamma=1$.

Table 1: Numerical errors and convergence orders in time direction with $\alpha=1.9, h=10^{-4}$

\begin{tabular}{ccccc}
\hline & \multicolumn{2}{c}{$\gamma=0$} & \multicolumn{2}{c}{$\gamma=1$} \\
\cline { 2 - 5 }$\Delta t$ & Error & Order & Error & Order \\
\hline $2^{-4}$ & $8.4209 e^{-2}$ & - & $4.7898 e^{-2}$ & - \\
$2^{-5}$ & $4.2197 e^{-2}$ & 0.9978 & $2.4132 e^{-2}$ & 0.9924 \\
$2^{-6}$ & $2.1129 e^{-2}$ & 0.9986 & $1.2134 e^{-2}$ & 0.9944 \\
$2^{-7}$ & $1.0525 e^{-2}$ & 1.0037 & $6.0529 e^{-3}$ & 1.0023 \\
\hline
\end{tabular}

Table 2: Numerical errors and convergence orders in time direction with $\alpha=1.9, \Delta t=10^{-4}$

\begin{tabular}{ccccc}
\hline & \multicolumn{2}{c}{$\gamma=0$} & \multicolumn{2}{c}{$\gamma=1$} \\
\cline { 2 - 5 }$h$ & Error & Order & Error & Order \\
\hline $2^{-4}$ & $6.1262 e^{-2}$ & - & $4.7052 e^{-2}$ & - \\
$2^{-5}$ & $3.1962 e^{-2}$ & 0.9583 & $2.4737 e^{-2}$ & 0.9510 \\
$2^{-6}$ & $1.6288 e^{-2}$ & 0.9811 & $1.2673 e^{-2}$ & 0.9759 \\
$2^{-7}$ & $8.1761 e^{-2}$ & 0.9961 & $6.3904 e^{-3}$ & 0.9916 \\
\hline
\end{tabular}

\section{Conclusion}

In this paper, an implicit finite difference method is developed for the one-dimensional space fractional wave equation with the Dirichlet and fractional boundary conditions. The unconditional stability of the method are proved with the Gerschgorin theorem and mathematical induction. The numerical experiment confirms the theoretical analysis and illustrates the practicability of the numerical scheme. Higher-order methods for the fractional wave equation will be considered in our future work.

\section{Acknowledgements}

This work was financially supported by the National Natural Science Foundation of China (11271141).

\section{References}

[1] R. Metzler and J. Klafter: Phys. Rep., Vol.339(1)(2000),p.1-77. 
[2] R. Metzler and J. Klafter: J. Phys. A: Math. Gen., Vol.37(31)(2004),p.R161-R208.

[3] F. Mainardi, M. Raberto, R. Gorenflo and E. Scalas: Physica A., Vol.287(2000),p.468-481.

[4] B.J. West, P. Grigolini and R. Metzler, and T.F. Nonnenmacher: Phys. Rev. E., Vol.55(1)(1997),p.99-106.

[5] A.V. Chechkin, V.Y. Gonchar, J. Klafter and R. Metzler: Advances in Chemical Physics, Vol.133 (B)(2006),p.439-496.

[6] A. De Pablo, F. Quirós, A. Rodriguez and J.L.Vázquez: Adv. Math., Vol.226(2)(2011),p. 1378-1409.

[7] A.Hussain and Q.A. Naqvi : Progress In Electromagnetic Research, Vol. 59(2006),p.199-213.

[8] Q.A. Naqvi, G. Murtaza and A.A. Rizvi: Optics Communications, Vol.178 (2000),p.27-30.

[9] A. Lakhtakia: Microwave Opt. Tech. Lett., 28(2001),p.385-386.

[10] A. Hussain, S.Ishfaq and Q.A.: Progress In Electro magnetics Research, Vol.63(2006),p.319-335.

[11] M.V. Ivakhnychenko, E.I. Veliev and T.V.: Journal of Electromagnetic Waves and Applications, Vol.21(13)(2007),p.1787-1802.

[12] K. Miller and B. Ross: An Introduction to the Fractional Calculus and Fractional Differential Equations( Wiley, New York, 1993).

[13] I. Podlubny: Fractional Differential Equations(Academic, 1999).

[14] S. Samko, A. Kilbas and O. Marichev: Fractional Integrals and Derivatives: Theory and Applications(Gordon and Breach, 1993).

[15] J. Jia, H.Wang: Journal of Computational Physics, Vol.293(2015),p.359-369.

[16] B.L. Guo, Q. Xu and Z. Yin: Applied Mathematics and Mechanics(English Edition), Vol.37(3)(2016),p.403-416.

[17] M.M. Meerschaert and C. Tadjeran: Journal of Computational and Applied Mathematics, Vol.172(2004),p.65-77.

[18] SG Samko, AA Kilbas, OI Marichev: Fractional Integrals and Derivatives: Theory and Applications (Gordon Breach, Yverdon, 1993).

[19] E. Isaacson and H.B. Keller: Analysis of Numerical Methods(Wiley, New York,1966).

[20] G.D. Smith: Numerical solution of partial differetial equations: Finite difference methods(Clarendon press, Oxford, 1985).

[21] D. Yu and H.Tan: Numerical methods of differetial equations(Science Publisher, Beijing, 2003.)

You and M. Kaveh: IEEE Trans. Image Process. Vol. 9(2000), p. 1723-1730. 